\title{
Parametric Studies of the R-Process in Supernova Shocks
}

\author{
Marius Eichler*i \\ Department of Physics, University of Basel, Klingelbergstrasse 82, 4056 Basel, Switzerland \\ E-mail: marius.eichler@unibas.ch
}

\section{Almudena Arcones}

Institut für Kernphysik, Technische Universität Darmstadt, Schlossgartenstrasse 2, D-64289

Darmstadt, Germany

E-mail: almudena.arcones@physik.tu-darmstadt.de

\section{Friedrich-Karl Thielemann}

Department of Physics, University of Basel, Klingelbergstrasse 82, 4056 Basel, Switzerland

E-mail: f-k.thielemann@unibas.ch

\begin{abstract}
We report a comprehensive study on the r-process in shocked surface layers. This astrophysical site was proposed by Ning et al. (2007). A sufficiently fast expansion can keep the neutron-to-seed ratio high up to the freeze-out of charged-particle reactions. Therefore, suitable conditions for the r-process are given even if matter is only slightly neutron-rich. The final abundances obtained with our model do not follow a uniform pattern and strongly depend on the initial conditions. In particular, the typical r-process peaks at $\mathrm{A}=130$ and 195 are suppressed. We study the impact of several input parameters on the outcome of the calculation and give insight into the nuclear reactions.
\end{abstract}

XII International Symposium on Nuclei in the Cosmos,

August 5-12, 2012

Cairns, Australia

\footnotetext{
${ }^{*}$ Speaker.

${ }^{\dagger}$ Support of the Swiss National Science Foundation and the NIC conference is acknowledged.
} 


\section{Introduction}

About half of the heavy elements $(\mathrm{A}>60)$ in our universe are produced by the rapid neutron capture process (or r-process). It is characterized by very fast neutron captures and subsequent $\beta$-decays, thus running through the extremely neutron-rich region of the nuclear chart.

Observations of r-process elements point towards a combination of several astrophysical sites, working at different times in the universe [1]. Shocked surface layers of O-Ne-Mg core-collapse supernovae may be one piece of the puzzle. It was shown that if the shock of an exploding $8.8 \mathrm{M}_{\odot}$ progenitor star can accelerate matter to high velocities $(\approx c / 2)$ r-process elements can be produced [2]. These low-mass stars have a steep density profile, which is why the shock accelerates to a very high velocity in the outer layers. The shell studied in Ref. [2] is at a radius of $1000 \mathrm{~km}$ where the progenitor composition is mainly dominated by carbon and oxygen. It was shown that a fast expansion leads to an early breakdown of NSE among neutrons, protons and ${ }^{4} \mathrm{He}$, maintaining a high neutron-to-seed ratio until the freeze-out of charged particle reactions [3].

However, recent supernova simulations $[4,5,6]$ did not support the assumptions made by [2]. In particular, they obtained lower values for the entropy and the shock velocity, also resulting in a different density and temperature.

\section{Method}

In a core-collapse supernova the supernova shock forms after collapse and propagates outwards through the star. We begin the description of a mass element at the encounter of the shock, followed by the expansion and cooling of the material. Since we aim for a broad study, we do not use data from supernova simulations, but employ a parametrized model for the evolution of the shocked material (a similar approach was used in e.g. [7]).

The initially free-falling mass shell is heated by the shock and expands outwards. For the description of the expansion we use two different approaches: The first follows the parametrization presented in Ref. [2]:

$$
\begin{gathered}
r(t)=r_{s}\left[1+\left(t / \tau_{1}\right)\right], \\
\rho(t)=\frac{\rho_{s}}{\left[1+\left(t / \tau_{1}\right)\right]^{2}\left[1+\left(t / \tau_{2}\right)\right]},
\end{gathered}
$$

In addition to this approach, we use a regular power-law expansion with a variable exponent $n$ which effects different expansion timescales:

$$
\begin{aligned}
& r(t)=r_{s}+v_{s}\left(t-t_{0}\right), \\
& \rho(t)=\rho_{s}\left(\frac{r_{s}}{r(t)}\right)^{n},
\end{aligned}
$$

where $t_{0}$ is the time of the shock passage and $r_{s}=r\left(t_{0}\right)$ is the radius of the mass shell at that time. The temperature is calculated by means of the Timmes \& Arnett (1999) Equation of State (EOS) [8]. 
For the nucleosynthesis calculations we use WINNET [10,11], a new extensive nuclear network, and the JINA reaction rate database ${ }^{1}$. The initial nuclear composition is given by the progenitor, as well as the density, pressure and temperature. If the temperature of the shocked matter exceeds $T_{N S E}=8 \mathrm{GK}$, NSE is assumed. It was shown that this assumption is reasonable even for fast expansions [12]. For lower temperatures the full network is solved.

At $T_{9} \approx 3$ the charged-particle reactions cease due to the Coulomb barrier, freezing the seed nuclei abundances. At that point, if there are enough free neutrons left, the neutron captures become dominant. Therefore, a high neutron-to-seed ratio at that time is crucial for the r-process.

\section{Results}

For our calculations we have used Nomoto's $8.8 \mathrm{M}_{\odot}$ progenitor [13] and studied the effect of different combinations of shock velocities, radii and after-shock expansions. The aim was to understand which conditions had to be fulfilled for the r-process to occur in the shocked surface layers. Section 3.1 deals with the identification of a favourable shock velocity and expansion. Later the results are examined more closely by focusing on the nuclear reactions in detail in section 3.2.

We performed our studies considering the thin $\mathrm{C}-\mathrm{O}$ layer surrounding the $1.38 \mathrm{M}_{\odot} \mathrm{O}-\mathrm{Ne}-\mathrm{Mg}$ core, located at $r \approx 10^{8} \mathrm{~cm}$ (the same layer that was studied in [2]). The density in this layer is between $10^{6}$ and $10^{5} \mathrm{~g} \mathrm{~cm}^{-3}$ and the temperature is $5.7 \times 10^{8} \mathrm{~K}$.

We find that the r-process can occur if two key conditions are fulfilled:

- high peak temperature, where the composition is determined by NSE

- fast expansion in order to prevent the consumption of neutrons before the charged-particle reaction freeze-out (see section 3.2)

In the shocked matter a peak temperature and density of $T_{9} \approx 9-10$ and $\rho_{6} \approx 2-3$ has to be reached $\left(\rho_{6}\right.$ is the density in units of $10^{6} \mathrm{~g} \mathrm{~cm}^{-3}$ ). This translates into an entropy of the order of 100. These conditions are found at a radius $r=10^{8} \mathrm{~cm}$.

\subsection{Dependence on Shock Velocity and Expansion}

The shock velocity determines the peak temperature and the dynamical expansion timescale. The latter constrains the key reactions which are those occurring faster than the expansion. In our case reactions destroying (or producing) neutrons are of special interest (see section 3.2). Thus, the neutron abundance and, accordingly, the neutron-to-seed ratio $\left(Y_{n} / Y_{\text {seed }}\right)$ are strongly influenced by the shock velocity.

In a first step we vary the shock velocity and use the expansion model employed in [2] (Eq. (2.1) \& (2.2)). The temperature evolution is calculated directly via the EOS in each timestep. The results are presented in Figure 1. The point $t=0$ is the time when the shock passes through the mass shell, so the figure only shows the expansion phase of the shocked matter.

\footnotetext{
${ }^{1}$ https://groups.nscl.msu.edu/jina/reaclib/db/
} 
The charged-particle reactions freeze out at $t \approx 2 \times 10^{-3} \mathrm{~s}$ when the temperature has dropped to 3 GK. The solid black line in Fig. 1 corresponds to the case investigated by [2] $\left(V_{s h}=1.5 \times 10^{10}\right.$ $\mathrm{cm} \mathrm{s}^{-1}$ ). In this calculation the peak temperature reaches a value of $T_{9}=9.92$. The initial neutron, proton and ${ }^{4} \mathrm{He}$ abundances are $Y_{n}=0.48, Y_{p}=0.48$ and $Y_{\alpha}=0.008$. These conditions lead to an rprocess and heavy nuclei are synthesized (see Fig. 1b). For slower shocks $Y_{n} / Y_{\text {seed }}$ is considerably lower throughout the expansion, which directly affects the final abundances.

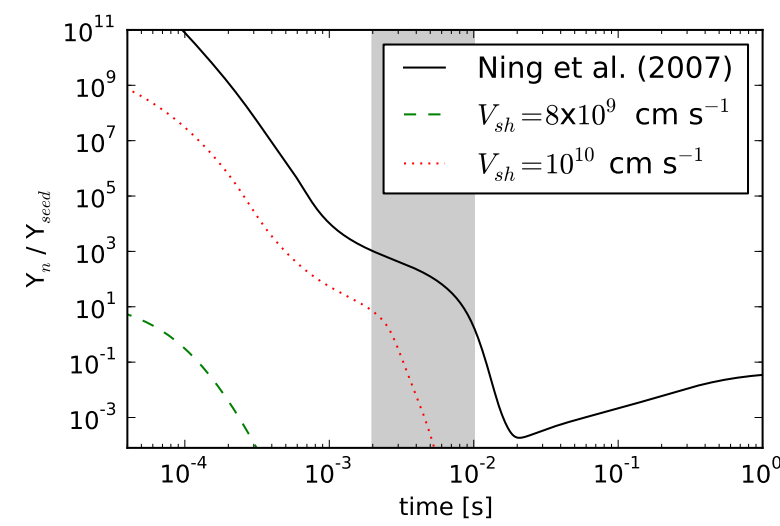

(a) neutron-to-seed ratio

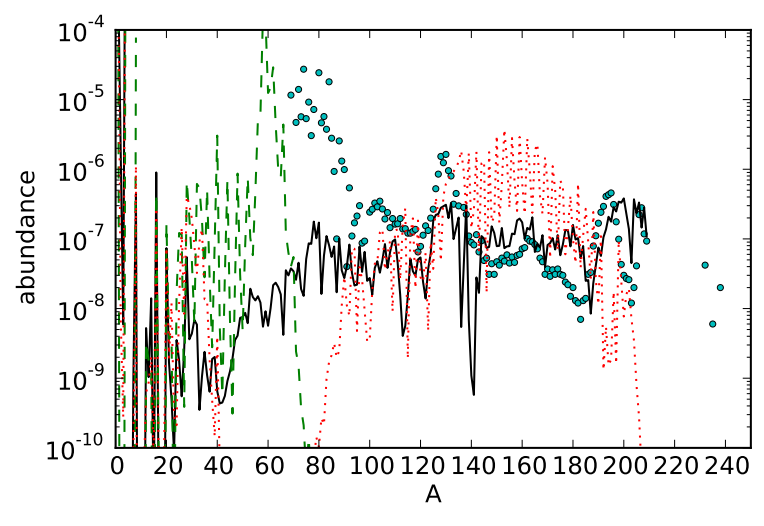

(b) final abundances

Figure 1: Neutron-to-seed ratio and final abundances for a set of calculations with different shock velocities using Nomoto's (1984) O-Ne-Mg progenitor. The initial shock radius is set to $R_{S h}=1000 \mathrm{~km}$ and the expansion follows the model of [2]. The black solid line corresponds to the case discussed in [2] $\left(V_{s h}=1.5 \times 10^{10} \mathrm{~cm} \mathrm{~s}^{-1}\right)$. Solar r-process abundances are represented by the blue dots (not to scale). The shaded area in 1a indicates the time after chargedparticle-reaction freeze-out when the temperature drops below $3 \mathrm{GK}$ (see text).

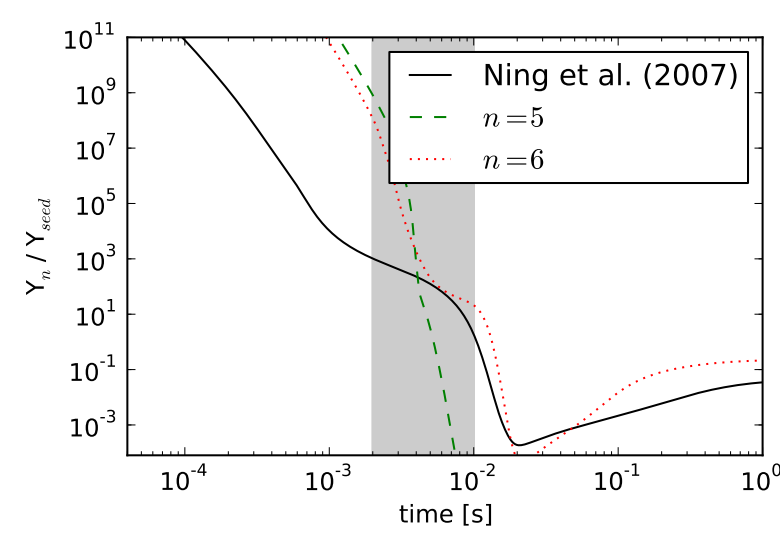

(a) neutron-to-seed ratio

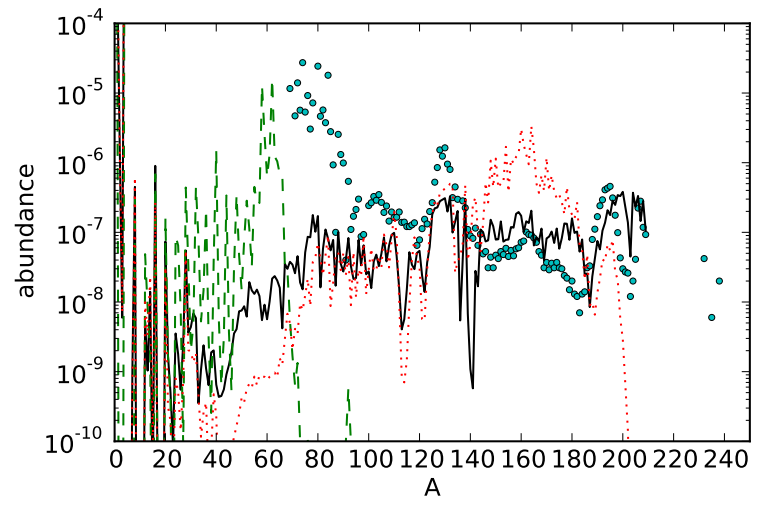

(b) final abundances

Figure 2: Results for a series of calculations with varying expansion and constant shock velocity $\left(V_{s h}=1.5 \times 10^{10} \mathrm{~cm}\right.$ $\mathrm{s}^{-1}$ and $R_{s h}^{\text {initial }}=1000 \mathrm{~km}$ ). The legend refers to the expansion models introduced in section 2 with the parameter $n$ the exponent in Eq. (2.4). 
In addition we have also studied the impact of different expansions for the same initial conditions (including $V_{s h}$ ). The results are presented in Figure 2. The expansion models follow the prescriptions introduced in section 2 and the parameter $n$ is the exponent in Eq. (2.4). In accordance with previous findings, an r-process occurs for expansions which are fast enough [3]. The threshold lies between $n=5$ and $n=6$. Similar to the cases with a fast shock velocity in Fig. 1 there is a $Y_{n} / Y_{\text {seed }}$ plateau around $4 \times 10^{-3} \mathrm{~s}$ for the faster expansions (Fig. 2a) which enables the synthesis of heavy nuclei (Fig 2b). The reasons for this will be discussed in section 3.2.

\subsection{Nuclear Reactions and Abundance Evolutions}

The expansion timescale affects the nuclear reaction rates that depend on the temperature. Therefore in a slower expansion those rates are higher than in a faster expansion at a given time. In our case the two most prominent reactions among the light nuclei are

$$
n+p \rightarrow D \quad \text { and } \quad n+D \rightarrow T
$$

As expected, both reactions are stronger in the slower expansion, consuming much more neutrons until they are virtually depleted at $t \approx 5 \times 10^{-3}$ s. This is shown in Figure 3, where the abundance evolutions of the light nuclei with mass numbers $A \leq 4$ are tracked for $n=5$ and $n=6$. In the slower expansion (Fig. 3a), the neutron, deuterium, tritium and ${ }^{3} \mathrm{He}$ abundances are depleted at around $5 \times 10^{-3} \mathrm{~s}$ as a consequence of the higher reaction fluxes. A comparison of Fig. 3 with Fig. 2a reveals that the difference in neutron abundance solely accounts for the difference in $Y_{n} / Y_{\text {seed }}$. Indeed, the seed abundances do not differ much in the two cases studied here.

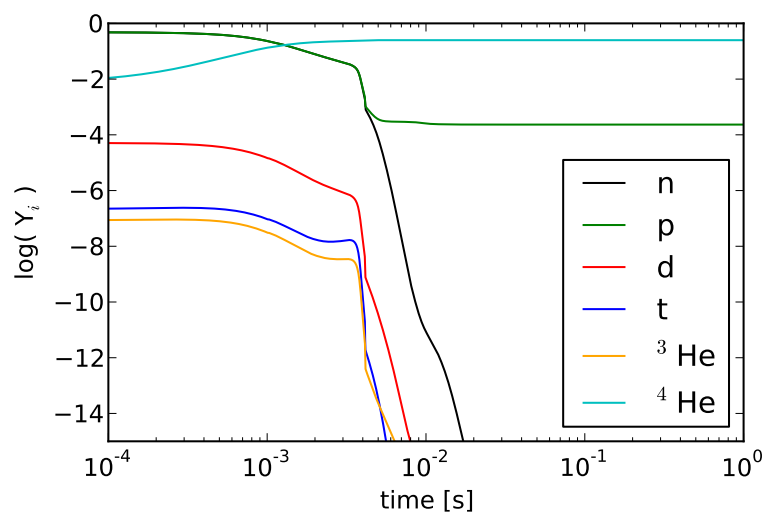

(a) $n=5$

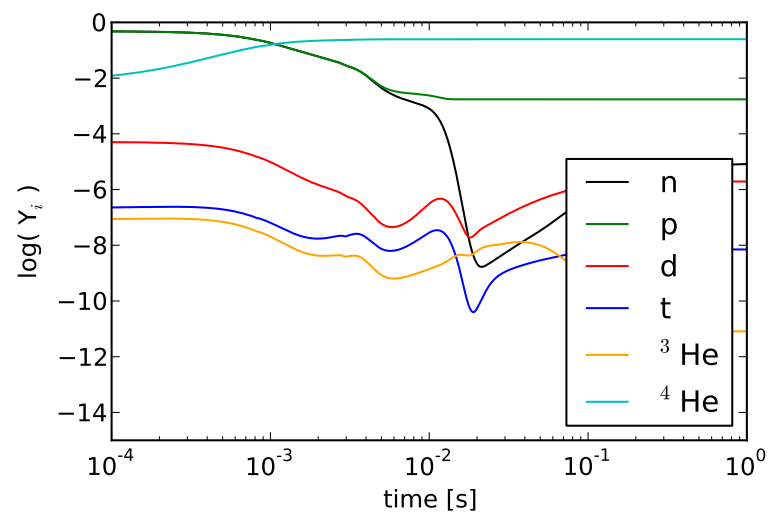

(b) $n=6$

Figure 3: Evolution of light nuclei abundances for an expansion with $n=5$ (a) and $n=6$ (b), corresponding to the trajectories in Fig. 2. From $4 \times 10^{-3}$ s onwards the abundances of all but the $\alpha$ particles diverge by several orders of magnitude. 


\section{Conclusions}

In this work we have presented a comprehensive study on the r-process in the environment of shocked surface layers, which have recently been proposed as an r-process site [2]. We have investigated the conditions that need to be fulfilled for the r-process and shown that, given those conditions, shocked surface layers could indeed contribute to the r-process abundances that are observed in the universe. Our calculations show that a peak temperature and density around $T=9-10 \mathrm{GK}$ and $\rho=2-3 \times 10^{6} \mathrm{~g} \mathrm{~cm}^{-3}$ combined with a rapid expansion provide favourable conditions for the production of r-process nuclei at a later time. We also have shown that the expansion timescale has a direct effect on the nuclear reactions and that in a slow expansion neutrons become depleted before the charged-particle reactions freeze-out.

Although the assumptions from NQM07 have been found to be too optimistic, new hydrodynamic supernova simulations will help to decide if any realistic models can meet the conditions we have identified.

\section{References}

[1] Y.-Z. Qian \& G. J. Wasserburg, Where, oh where has the r-process gone?, 2007, PhysRep, 442, 237

[2] H. Ning, Y.-Z. Qian, \& B. S. Meyer, r-Process Nucleosynthesis in Shocked Surface Layers of O-Ne-Mg Cores, 2007, ApJL, 667, L159

[3] B. S. Meyer, r-Process Nucleosynthesis without Excess Neutrons, 2002, Physical Review Letters, 89, 231101

[4] H.-T. Janka, B. Müller, F. S. Kitaura \& R. Buras, Dynamics of shock propagation and nucleosynthesis conditions in O-Ne-Mg core supernovae, 2008, A\&A, 485, 199

[5] R. D. Hoffman, B. Müller, \& H.-T. Janka, Nucleosynthesis in O-Ne-Mg Supernovae, 2008, ApJL, 676, L127

[6] S. Wanajo, K. Nomoto, H.-T. Janka, F. S. Kitaura, \& B. Müller, Nucleosynthesis in Electron Capture Supernovae of AGB Stars, 2009, ApJ, 695, 208

[7] W. A. Fowler \& F. Hoyle, Neutrino Processes and Pair Formation in Massive Stars and Supernovae, 1964, ApJS, 9, 201

[8] F. X. Timmes \& D. Arnett, The Accuracy, Consistency, and Speed of five Equations of State for Stellar Hydrodynamics, 1999, ApJS, 125, 277

[9] A. Arcones, H.-T. Janka, \& L. Scheck, Nucleosynthesis-relevant conditions in neutrino-driven supernova outflows. I. Spherically symmetric hydrodynamic simulations, 2007, A\&A, 467, 1227

[10] C. Winteler, R. Käppeli, A. Perego, et al., Magnetorotationally Driven Supernovae as the Origin of Early Galaxy r-Process Elements?, 2012, ApJL, 750, L22

[11] C. Winteler, Light Element Production in the Big Bang and the Synthesis of Heavy Elements in 3D MHD Jets from Core-Collapse Supernovae, 2011, PhD Thesis, University of Basel

[12] C. Freiburghaus, J.-F. Rembges, T. Rauscher, et al., The Astrophysical r-Process: A Comparison of Calculations following Adiabatic Expansion with Classical Calculations Based on Neutron Densities and Temperatures, 1999, ApJ, 516, 381

[13] K. Nomoto, Evolution of 8-10 solar mass stars toward electron capture supernovae. I - Formation of electron-degenerate $O+N E+M G$ cores, 1984, ApJ, 277, 791 